\title{
On the Separation of Quantum Noise for Cardiac X-Ray Image Compression
}

\author{
Frederik J. de Bruijn and Cornelis H. Slump \\ Dept. of Electrical Engineering, Laboratory for Network Theory and VLSI-Design, \\ University of Twente, P.O. Box 217, 7500 AE Enschede, The Netherlands \\ F.J.deBruijn@el.utwente.nl
}

\begin{abstract}
In lossy medical image compression, the requirements for the preservation of diagnostic integrity cannot be easily formulated in terms of a perceptual model. Especially, since human visual perception is dependent on numerous factors such as the viewing conditions and psycho-visual factors. Therefore, we investigate the possibility to set criteria for data loss, based on the characteristics of the acquisition system. In general, medical images tend to be relatively noisy, due to the low patient dose. The nature of the noise is usually known and partially uncorrelated. In addition, the systems modulation transfer function (MTF) imposes a known spatial-frequency limitation to the information. In the assumption that noise which is not correlated with the signal contains no diagnostic information, we derive compression criterion based on a physical model of a digital cardiac cineangiography system.
\end{abstract}

\section{INTRODUCTION}

An important issue in lossy compression of medical images is the risk of destroying diagnostically relevant information. Current lossy compression standards, such as JPEG and MPEG, are designed for conventional still-image and video display. These algorithms are intended for viewing situations in which loss of image detail is not regarded as a loss of information to the observer, but is primarily assessed in an aesthetical sense. The data omission in conventional lossy image compression is mostly based on commonly accepted models of human visual perception [1], [2].

Some successful attempts have been reported of the application of perceptive criteria in lossy medical compression [3], [4]. It is debatable, however, whether perceptive criteria are sufficient for preservation of diagnostic information.

In this paper, we derive a compression criterion which is not based on properties of the human observer, but on limitations of the image acquisition process, in which noise plays a key-role. The paper focuses on a cardiac X-ray imaging system, using an image intensifier-TV system.

\section{THEORY}

Discussing the image statistics of cardiovascular X-ray images, we can distinguish two statistics: the statistics of the medical data and the statistics due to the acquisition process.

The statistics of cardiovascular images fit very well to a first-order Markov-process [4]. In addition, the quantum noise due to the detection process can be modeled by a Poisson distribution of which the mean value is determined by the photon flux detected at the entrance screen. The total is low-pass filtered by the MTF of the system. Clearly, due to the Poisson nature, the quantum noise is correlated with the medical data.

We assume that, in the hypothetical case of an additional Gaussian noise component which is not correlated with the signal, the noise contains no diagnostic information. In a spectral description, any component solely associated with the noise can be omitted in compression (and possibly regenerated for perceptual motives) [5]. In order to delete the diagnostically irrelevant components, we seek a description of the acquired data in a form of which both statistics can be observed separately, preferably by creating two independent stochastic signals.

\section{Filtered Poisson noise process}

The number of detected photons per pixel at location $(x, y)$ is a random variable $\mathbf{n}(x, y)$, which is a matrix with Poisson distributed data with mean $\lambda_{\mathbf{n}}(x, y)$, corresponding to the mean photon-flux variation. For clarity, we observe the one-dimensional signal $\mathrm{n}(x)$ and simplify the analysis by considering a constant mean $\lambda_{\mathbf{n}}(x)=\lambda_{\mathbf{n}}$

$$
P(\mathbf{n}(x)=n)=\frac{\lambda_{\mathbf{n}}^{n}}{n !} e^{-\lambda_{\mathbf{n}}}
$$

A signal $\mathbf{f}$ is formed by convolution with $\mathbf{h}$, the impulse response associated with the MTF. The signal $f$ is a process known as shot noise with mean $\mu_{\mathrm{f}}$ and variance $\sigma_{\mathrm{f}}^{2}$,

$$
\begin{gathered}
\mathbf{f}=\mathbf{h} * \mathbf{n}, \quad \text { with } \\
\mu_{\mathbf{f}}=\lambda_{\mathbf{n}} \int_{-\infty}^{\infty} \mathbf{h}(x) d x, \quad \sigma_{\mathbf{f}}^{2}=\lambda_{\mathbf{n}} \int_{-\infty}^{\infty} \mathbf{h}^{2}(x) d x
\end{gathered}
$$

according to Campbell's theorem [6].

\section{Non-linear transformation}

In [7], Anscombe showed that, if $\mathbf{n}$ is a Poisson distributed variable with mean $\lambda_{n}$, the transformation

$$
\mathbf{g}=T(\mathbf{n})=\sqrt{\mathbf{n}+c},
$$

with $c=\frac{3}{8}$, results in a variable $\mathrm{g}$ which is normally distributed with a nearly constant variance $\sigma_{\mathrm{g}}^{2}=\frac{1}{4}$ already for moderate values of $\mathbf{n}$. As $\lambda_{\mathbf{n}}$ is large, we set $c$ equal to zero.

To evaluate the suitability of transformation (4) to a filtered Poisson process, we assume that $\lambda_{\mathbf{n}}$ is large enough to approximate both the Poisson process $\mathbf{n}$ and filtered Poisson process $\mathbf{f}$ by a Gaussian noise process. For $\mathbf{n}$, mean and variance are equal, $\mu_{\mathbf{n}}=\lambda_{\mathbf{n}}$ and $\sigma_{\mathbf{n}}^{2}=\lambda_{\mathbf{n}}$ respectively. For $\mathbf{f}$, however, mean and variance differ by a factor $\beta$ which is independent of $\lambda_{\mathbf{n}}$

$$
\sigma_{\mathbf{f}}^{2}=\beta \mu_{\mathbf{f}}, \quad \text { with } \quad \beta=\frac{\int_{-\infty}^{\infty} \mathbf{h}^{2}(x) d x}{\int_{-\infty}^{\infty} \mathbf{h}(x) d x} .
$$


In order to apply (4) to the filtered process $f$, mean and variance need to be equal. By correcting $f$ with a factor $\frac{1}{\beta}$, a signal $\mathbf{k}$ is obtained with equal mean and variance,

$$
\mathbf{k}=\frac{\mathbf{f}}{\beta}, \quad \text { with } \quad \mu_{\mathbf{k}}=\frac{\lambda_{\mathbf{n}}}{\beta}, \quad \sigma_{\mathbf{k}}^{2}=\frac{\lambda_{\mathbf{n}}}{\beta} .
$$

Hence, transformation of $\mathbf{k}$ with expression (4) results in a process $\mathbf{g}$,

$$
\mathbf{g}=\sqrt{\mathbf{k}}=\sqrt{\frac{\mathbf{f}}{\beta}}, \text { with } \mu_{\mathbf{g}}=\sqrt{\frac{\lambda_{\mathbf{n}}}{\beta}}, \sigma_{\mathbf{g}}^{2}=\frac{1}{4} .
$$

Noise power spectrum

After convolution with $\mathbf{h}$ and correction with factor $\beta$, a signal $\mathbf{k}$ is obtained with autocorrelation function

$$
\begin{aligned}
\mathbf{R}_{\mathbf{k k}}(\xi) & =\frac{\lambda_{\mathbf{n}}}{\beta^{2}}(\mathbf{h} * \mathbf{h})(\xi)+\frac{\lambda_{\mathbf{n}}^{2}}{\beta^{2}} \mathbf{H}^{2}(0) \\
& =\sigma_{\mathbf{k}}^{2} \rho(\xi)+\mu_{\mathbf{k}}^{2} \mathbf{H}^{2}(0),
\end{aligned}
$$

where $\boldsymbol{\rho}(\xi)=\frac{1}{\beta}(\mathbf{h} * \mathbf{h})(\xi)$ is a normalized correlation coefficient.

By approximating the joint Poisson density by a joint normal density to determinate the autocorrelation [6], and by local linearization of transformation $T$, the autocorrelation of $\mathbf{g}$ can be approximated by

$$
\mathbf{R}_{\mathrm{gg}}(\xi)=\frac{1}{4} \rho(\xi)+\sqrt{\mu_{\mathbf{k}}^{2} \mathbf{H}^{2}(0)}
$$

which results in a noise power spectrum which is independent of the mean photon count $\lambda_{\mathbf{n}}$,

$$
\mathrm{S}_{\mathrm{gg}}(\omega)=\frac{1}{4} \frac{1}{\beta} \mathbf{H}^{2}(\omega)+2 \pi \frac{\lambda_{\mathbf{n}}}{\beta} \mathbf{H}^{2}(0) .
$$

\section{APPLICATION TO A CARDIAC IMAGING MODEL}

The low-pass filtering by the total acquisition chain is described by a modulation transfer function (MTF). The following expression can be used as a model for an MTF with circular symmetry

$$
\mathbf{H}(\omega)=\alpha \operatorname{MTF}(\omega)=\alpha e^{-\left(\frac{\omega}{\omega_{c}}\right)^{2}},
$$

with $\omega$ the radial spatial frequency, $\omega_{c}$ a frequency scaling parameter, and $\alpha$ a factor describing the overall gain of the system [8], [9].

With (11) and (10), the power spectrum becomes

$$
\mathrm{S}_{\mathbf{g g}}(\omega)=\frac{1}{4} \frac{\alpha^{2}}{\beta} e^{-2\left(\frac{\omega}{\omega_{c}}\right)^{2}}+2 \pi \frac{\lambda_{\mathbf{n}} \alpha^{2}}{\beta}, \text { with } \beta=\frac{\alpha \omega_{c}}{2 \sqrt{2 \pi}} .
$$

A practical value of the frequency scaling parameter is $\omega_{c}=$ $1.77[10]$. The corresponding normalized spectrum is depicted in Figure 1.

Experiments have shown that cardiovascular images can be modeled by a first-order Markov process with the correlation coefficient $\rho=0.99$ [4], which seems to be in agreement with the value reported in [3]. The power spectrum of a first-order Markov process [11] is given by

$$
\mathrm{S}(\omega)=\frac{1-\rho^{2}}{1-2 \rho \cos \omega+\rho^{2}} .
$$

Figure I shows how tightly the signal energy is packed into the low frequency components, compared to the noise power spectrum. This may indicate the potential of this transformation in the view of lossy compression. Practical experi-

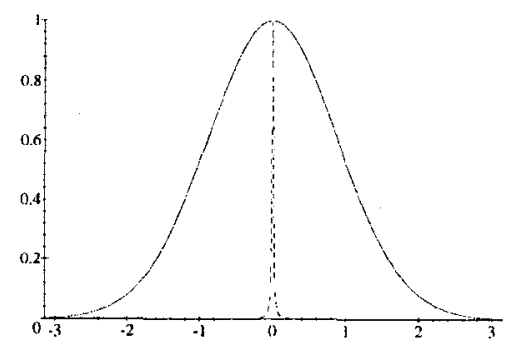

Fig. 1. Comparison power spectra: normalized noise power spectrum for $\omega_{c}=1.77$ (solid), normalized signal power spectrum for $\rho=0.99$ (dashed).

ments [5] indicate that the signal-to-noise ratio is such that a separation between the signal components and the quantumnoise components is possible.

\section{CONCLUSION}

We have determined an expression which transforms a filtered Poisson-distributed signal into a 'noise-free' signal term and a noise term, uncorrelated with the signal. In addition, an expression for the noise power spectrum of the transformed signal has been derived, which is based upon a model of a cardiac imaging system. In case the acquisition parameters are known, the true amplitude of the noise power spectrum can be estimated.

\section{REFERENCES}

[1] J. L. Mannos and D. J. Sakrison, "The effects of a visual fidelity criterion on the encoding of images," IEEE Transactions on Information Theory, vol. 20, pp. 525-535, July 1974.

[2] N. B.Nill, "A visual model weighted cosine transform for image compression and quality assement," IEEE Transactions on Communications, vol. 33, pp. 551-557, June 1985.

[3] M. Breeuwer, R. Heusdens, and P. Zwart, "Overlapped transform coding of medical X-ray images," in Medical Imaging 1994: Image Caplure, Formatting, and Display (Y. Kim, ed.). vol. 2194, pp. 264-275. SPIE, Feb. 1994

[4] P. Hagendoorn. "Compression of medical X-ray images while preserving diagnostic value," Master's thesis, University of Twente, Dept. of Electrical Engineering, Enschede, The Netherlands, Dec. 1995. Reportcode EL-BSC-094N95.

[5] C. J. E. van Heerde, "Reductie van ruis in coronaire angiogrammen door $\ell_{2}$-compressie," Master's thesis. University of Twente, Dept. of Elcctrical Engineering, Enschede, The Netherlands, Feb. 1996. 250hours assignment, Reportcode EL-BSC-007N96 (in Dutch).

[6] A. Papoulis, Probability, Random Variables, and Stochastic Processes. McGraw-Hill Series in Electrical Engineering, McGraw-Hill, 2nd. ed., 1984.

[7] F. Anscombe, "The transformation of poisson, binomial and negativebinomial data," Biometrika, vol. 35, pp. 246-254, 1948.

[8] H. E. Johns and J. R. Cunningham, The Physics of Radiology. Springfield, IL: Charles C. Thomas, 4 ed.. 1983.

[9] L. A. J. Verhoeven, Digital Subtraction Angiography: The Technique and an Analysis of the Physical Factors Influencing the Image Quality. PhD thesis, Technische Hogeschool Delft, Delft, The Netherlands, Jan. 1985.

[10] M. Brok and C. H. Slump, "Automatic determination of image quality parameters in digital radiographic imaging systems," in Medical Imaging III: Inage Formation (R. H. Schneider et al, eds.), vol. 1090, pp. 246-256, SPIE, 1989.

[11] T. Berger, Rate Distortion Theory: A Mathematical Basis for Data Compressiom. Electrical Engineering Series, Information and System Sciences Series, Englewood Cliffs, NJ: Prentice-Hall, Inc., 1971. 